\title{
Broad-band dispersion measurement of ZBLAN, germanate and silica fibers in mid-IR
}

\author{
D. Klimentov, N. Tolstik, V.V. Dvoyrin, V.L. Kalashnikov and I. T. Sorokina
}

\begin{abstract}
We report the first ultra-broad band dispersion measurements in short-length ZBLAN, germanate and silicabased optical fibers in the near- and mid-IR wavelength ranges between 1.7 and $2.0 \mu \mathrm{m}$ and from 2.3 to $2.45 \mu \mathrm{m}$, using two ultrabroadband light sources: a broadband superluminescent Tmdoped fiber source and a novel femtosecond pulsed mode-locked Cr:ZnS oscillator. The measured second order dispersion characteristics of the fibers correspond to the theoretical predictions (numerical calculations).
\end{abstract}

Index Terms - Dispersion, Lasers, Measurement techniques, Optical fibers.

\section{INTRODUCTION}

$\mathrm{D}^{-1}$ UE to the rapid development of laser technology in the wavelength region around $2 \mu \mathrm{m}$, and in particular, development of pulsed fiber lasers and laser systems, the control and compensation of group-velocity dispersion (GVD) in that wavelength range is an important task. This is especially essential for development of ultrashort pulse laser systems in mid-IR. For such systems we have to precisely control the dispersion effect that requires accurate measurements of the dispersive properties of optical fibers in a wide spectral region. Precise knowledge of GVD in novel nonlinear and various specialty fibers in mid-IR is important to be able not $o^{1}$ nly to build an ultrafast oscilator, but also to produce a white-light continuum from these laser sources. Measurement of dispersion at and especially above $2 \mu \mathrm{m}$ is a challenging task because of the lack of available commercial broadband light sources in this wavelength region, required for measurement. At the moment, there is a growing interest in dispersion measurements in mid-IR range that is illustrated by recent publications and conference reports, in particular, the first measurements of ZBLAN fibers have been reported in [1], [2].

Due to the chromatic-dispersion effects, or group-velocity dispersion, different wavelengths propagate with the different group velocities, causing broadening of short optical pulse and nonlinear optical distortion. Several techniques have been

\section{ACKNOWLEDGMENT}

This work is supported by the Norwegian Research Council (NFR) projects FRITEK/191614, MARTEC MRL. We also would like to acknowledge IR Photonics FORC for providing the ZBLAN fibers and germanate fibers correspondingly. developed to measure the dispersion of optical fibers [3]-[5], such as: time-of-flight, phase-shift, interferometric, or degenerate-four-wave-mixing-based techniques. For time-offlight methods a source of narrow light pulses covering a wide spectral region is required. Discrete-wavelength laser diodes [6] and broadband sources such as fiber Raman lasers [7], [8], supercontinuum sources [9]-[11], or tunable soliton sources [12] are commonly used for this. The output of such broadband sources can cover several hundreds of nanometers and even more. The phase-shift technique is based on the phase delay measurements of a pulsed or amplitude modulated signal as a function of wavelength. But these techniques were developed for long optical fiber samples and the fiber length of several kilometers is required. However, it is often difficult to provide such long fiber lengths for dispersion measurement, especially in case of new types of specialty fibers.

For the dispersion measurement of short-length fibers, interferometric technique is normally used [13], where a sample fiber is placed in one arm of an interferometer, and the air gap is used as the reference arm.

The broad-band interferometric dispersion measurements in short-length fibers were mostly performed with LEDs producing spectral coverage $\Delta \lambda$ of 50 to $100 \mathrm{~nm}$ [14], [15], which require a wide range of diode sets or diode lines; or amplified spontaneous emission (ASE) sources producing $\Delta \lambda$ of 100 to $200 \mathrm{~nm}$ [16]; or low-intensity white-light sources [17]. In the last two cases amplification of the signal is necessary, but the measurement accuracy is still limited, because, with the signal amplification, the noise is also amplified. As a result, these measurements were characterized by low signal/noise $(\mathrm{s} / \mathrm{n})$ ratio.

In this work we present a novel setup for mid-IR measurements of dispersion parameter in optical fibers in two wavelength ranges, 1.7 to 2.0 and 2.3 to 2.45 microns; and results of dispersion measurements of specialty short-length fibers for infrared applications with a resolution up to $20 \mathrm{~nm}$. The setup is distinguished from the existing similar measurement systems [16], [18] by using new broadband light sources, such as superluminescent Tm-doped fiber based source and a recently developed in our lab mode-locked femtosecond Cr:ZnS oscillator [19], [20]. These sources allow achieving broad spectral coverage with the higher output power and with a high signal/noise ratio. To the best of our knowledge, it is the broadest direct dispersion measurement in mid-IR (above $2 \mu \mathrm{m}$ ) and the broadest with the ASE source.

In this paper, as a demonstration of capabilities of the setup, we present dispersion measurements of specialty fibers, such 
as: the highly-germanium-doped core fiber co-doped with thulium, soft glass step-index ZBLAN fiber from IR Photonics, and standard silica telecommunication fibers of the SMF-28 type for cross-check.

\section{EXPERIMENTAL SETUP}

The interferometric setup (Fig. 1.) was developed for dispersion measurements in single-mode optical fibers with a length of $0.6-2.5 \mathrm{~m}$. The setup is based on Mach-Zehnder interferometer. The dispersion of several short-length singlemode fibers was measured. Namely, we studied the highlygermanium-doped core fiber co-doped with Thulium [21] with an approximately $3 \mu \mathrm{m}$ core diameter, soft glass step-index ZBLAN fiber with a $10.5 \mu \mathrm{m}$ core and a numerical aperture of 0.17 , and standard telecommunication fibers to confirm the capabilities of our setup.

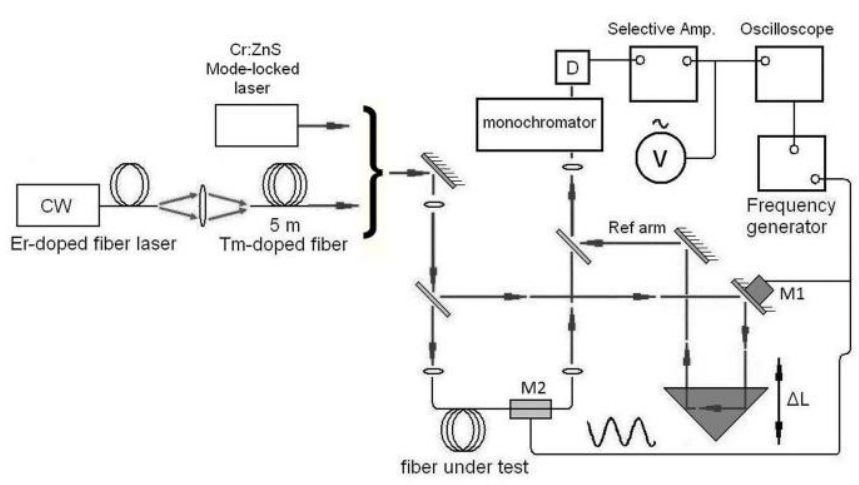

Fig. 1. The experimental setup for broad-band dispersion measurements of short-length fibers.

In order to cover the wavelength ranges of the interest, two broadband light sources were used for interferometer. For the first region the fiber ASE source has been specially developed. For this purpose, a high numerical aperture $5 \mathrm{~m}$ long Tmdoped fiber was pumped at $1.61 \mu \mathrm{m}$ wavelength by $\mathrm{CW}$ Erdoped fiber laser with the power of $400 \mathrm{~mW}$, producing a 300 $\mathrm{nm}$ broad (50 nm FHWM) superluminescent emission around $1.82 \mu \mathrm{m}$. To prevent lasing in Tm-doped fiber, its output end was cleaved to 7 degrees. The corresponding superluminescence spectrum is shown below (Fig. 2.).

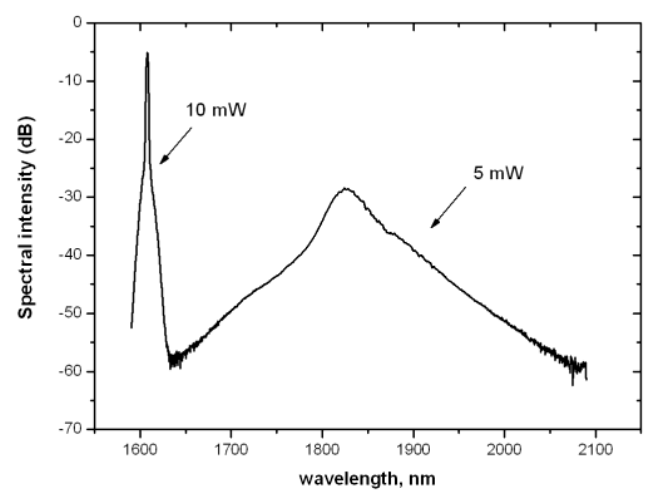

Fig. 2. The superluminescence spectrum of $5 \mathrm{~m}$ long Tm-doped fiber used for pumping the experimental setup.

The optical power of the ASE reached $5 \mathrm{~mW}$. This power level exceeds, to our knowledge, the optical power of the best commercially available superluminescent semiconductor lasers in this wavelength range.

For longer wavelength region of 2.3 to $2.45 \mu \mathrm{m}$, for the first time, we used the mode-locked femtosecond $\mathrm{Cr}: \mathrm{ZnS}$ oscillator [20]. The laser was pumped by the $1.61 \mu \mathrm{m}$ Er-fiber laser from IPG Photonics produced up to $5 \mathrm{~W}$ of optical power. It produced pulses of about $70 \mathrm{fs}$ at the central wavelength of $2.39 \mu \mathrm{m}$, with the repetition rate of $150 \mathrm{MHz}$ and output power up to $550 \mathrm{~mW}$. The emission spectrum of the laser reached $100 \mathrm{~nm}$ FWHM (Fig. 3.). It is the broadest spectrum obtained from the femtosecond $\mathrm{Cr}: \mathrm{ZnS}$ laser in this wavelength range.

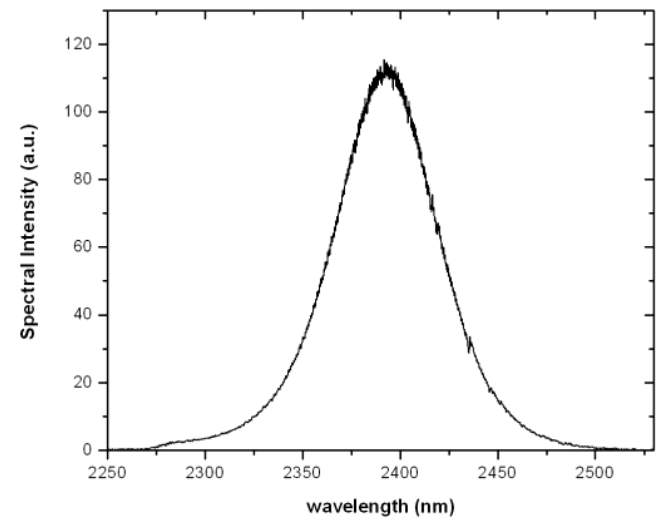

Fig. 3. The emission spectrum of $\mathrm{Cr}: \mathrm{ZnS}$ laser used for pumping the experimental setup.

The fiber under test was placed into the sample arm of the interferometer, and the air arm was used as the reference arm with variable length. For coupling and decoupling we used 50/50 dielectric beamsplitters. For phase modulation we used two types of modulators, "M1" and "M2", depending on type of the fiber coating (bare fiber or patch cord). For phase modulation of fiber patch cord we used an ordinary audio speaker with a glued mirror ("M1"), placed into the reference arm to periodically change its length. As the phase modulator "M2" we used the electromagnetic fiber phase modulator. Modulation of the beam phase in the fiber under test occurred due to the induced longitudinal tension of the fiber, providing the change of optical path up to several wavelengths. The phase modulators, "M1" and "M2", were connected to the frequency generator and operated at about 300 and $190 \mathrm{~Hz}$ frequency respectively. The spectral selection and detecting were performed by double-prism Carl Zeiss monochromator and a sensitive extended InGaAs Hamamatsu photodetector.

The best interference signal on the photodetector for a fixed wavelength corresponds to the equal arm lengths alignment. In the experiment we measured the dependence of the change in the length of the reference arm required to equalize it with 
beam path in the sample arm on the wavelength. The signal from the detector was directed to selective amplifier to distinguish the interference signal from the noise and then to $\mathrm{AC}$ millivoltmeter. The use of such low noise measurement technique with bright source allows achieving the $s / n$ ratio of about 7 at $1820 \mathrm{~nm}$ wavelength and 2 at the edges of the spectral range of the superluminescent source. At the same time, we controlled the shape of the interference signal on the oscilloscope. Based on these data, the dependence of the path difference $\Delta \mathrm{l}$ on the wavelength $\lambda$ was plotted. Dispersion parameter $\mathrm{D}(\lambda)$ and the path difference $\Delta \mathrm{l}(\lambda)$ are related through (1)[5]:

$$
D(\lambda)=\frac{1}{c L} \frac{d[\Delta l(\lambda)]}{d \lambda}
$$

where $L$ is the length of the fiber under test, $c$ is the speed of light.

\section{RESULTS AND DISCUSSION}

For calibration of our setup two different fibers similar to SMF-28 were studied. The first one - a standard telecommunication fiber widely available at the market and other one, silica fiber with the similar chemical composition, that was drawn at our lab at NTNU. The dispersion parameter was measured in the range from 1.7 to $2.0 \mu \mathrm{m}$ and from 2.3 to $2.45 \mu \mathrm{m}$ (Fig. 4.) and compared with calculation of dispersion performed in Fiber Optics Research Center (FORC), Russian academy of sciences as well as our own calculations.

The results of measurement and the calculation data showed a good agreement with each other thus indicating the reliability of our measurements. This is illustrated in more detail in the inset (Fig. 4.) for shorter wavelength region. The results obtained with both fibers were similar, that also indicates a good quality of the fiber drawn at NTNU.

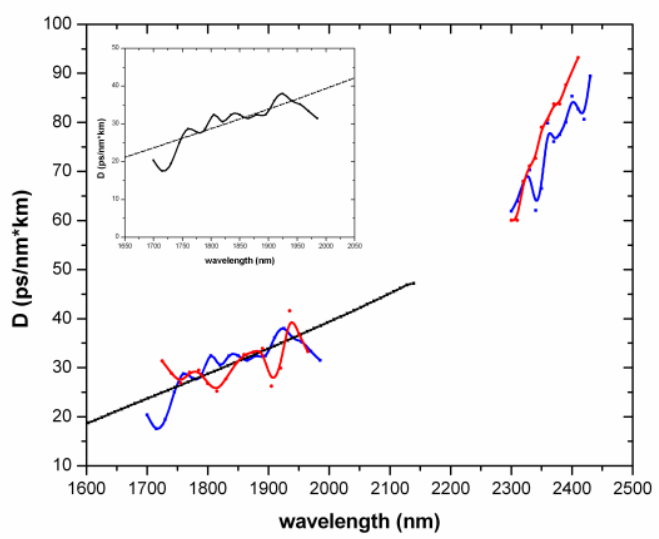

Fig.4. The results of dispersion measurements in the silica single-mode fibers (blue and red lines, red - drawn at NTNU, blue - commercial fiber); in comparison with the calculation data from FORC (dashed line). The inset shows shorter wavelength region in more detail.
The results of dispersion measurements in the highlygermanium-doped core fiber co-doped with thulium are shown at the Fig. 5. The length of the tested fiber was $80 \mathrm{~cm}$. The fiber was single-mode with a cut-off at $1.43 \mu \mathrm{m}$. It showed anomalous dispersion at our wavelengths of interest in contrast to the standard silica fibers in Fig. 4.

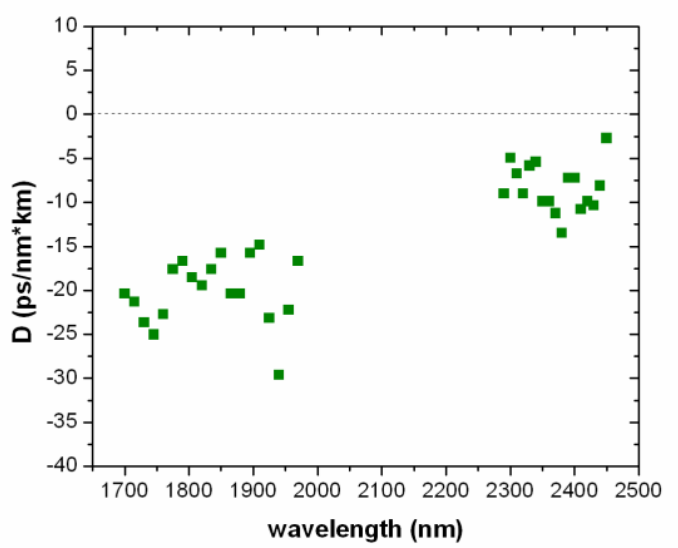

Fig. 5. The results of dispersion measurements in highly-Ge-doped fiber codoped with Tm.

The results of measurements of dispersion parameter in ZBLAN fibers were already reported in [2] for the wavelength range of $0.9-1.6 \mu \mathrm{m}$. In this paper, we present the results of dispersion measurements and theoretical calculations in such fibers for the longer wavelength range of 1.6 to $2.45 \mu \mathrm{m}$ (Fig. 6.). The results were obtained with the mirror modulator. The length of tested fiber was $210 \mathrm{~cm}$. The calculated and measured zero-dispersion wavelengths are similar; the measured one equals to $1.70 \mu \mathrm{m}$ and the calculated one equals to $1.71 \mu \mathrm{m}$. 

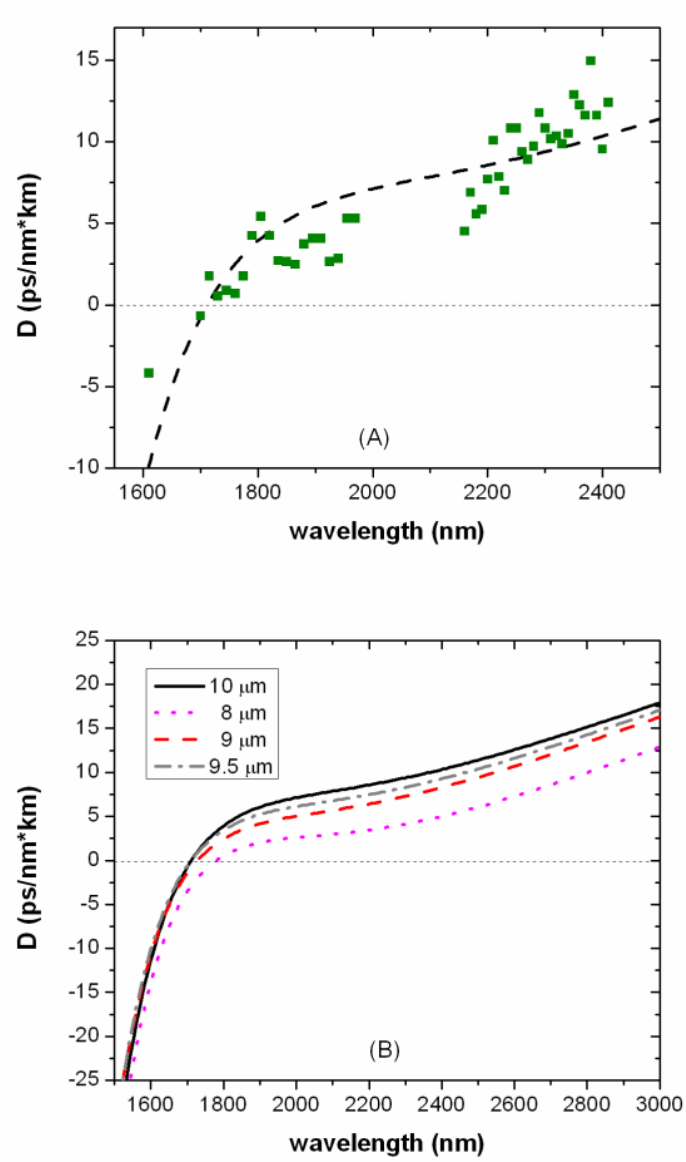

Fig. 6. The results of the dispersion measurements (green) in comparison with the theoretical calculation (black dashed) for soft glass

step-index ZBLAN fiber with a $10.5 \mu \mathrm{m}$ core diameter (a); and theoretical calculations of dispersion parameter in ZBLAN fiber with a different core diameters (b).

The calculation of dispersion parameter has been based on the finite-element vectorial modelling of an effective refractive index of zero-order mode by the means of the COMSOL FEMLAB software package in combination with the Lumerical's MODE Solutions package. The wavelength dependence of the bulk material refractive index for different core diameters has been approximated by Sellmeier's formula in correspondence with [22]. The obtained solutions have been compared with the analytical results for a step-index ZBLAN fibers. The corresponding Maple computer algebra introduction can be found in [23]. The results of the measurements match theoretical estimation (Fig. 6.) based on ZBLAN Sellmeier data.

Possible sources of errors in our measurements were: dispersion of optical objectives and lenses in the sample arm which supposed to be negligible; presence of water absorption in the air parts of the interferometer arms which also was not taken into account. These factors were omitted during the analysis, because the total thickness of the optical elements mentioned before was negligible as compared to the optical path in the interferometer arms; there was no correspondence between features in the measured spectra and the relevant spectral absorption lines of water.

\section{CONCLUSION}

In conclusion, in this paper a novel interferometric setup for measurement of dispersion of specialty short-length fibers for infrared applications was developed, tested and used for the investigation of the new fibers in the previously hardly accessible wavelength range around and above $2 \mu \mathrm{m}$. The characteristic feature of the setup is that it is based on the two novel broadband sources: rather intensive superluminescent Tm-fiber source and a novel femtosecond pulsed mode-locked $\mathrm{Cr}: \mathrm{ZnS}$ oscillator allowing to cover almost the whole wavelength range of 1.7 to $2.45 \mu \mathrm{m}$ with the resolution of measurements up to $20 \mathrm{~nm}$. The similar approach is applicable for the development of the setup for dispersion measurement in different spectral ranges, in particular, in the range near 3 $\mu \mathrm{m}$, which is in progress now.

\section{REFERENCES}

[1] C. Agger, C. Petersen, S. Dupont, H. Steffensen, J. K. Lyngsø, C. Thomsen, S. Keiding, and O. Bang, "ZBLAN supercontinuum generation - detailed comparison between measurement and simulation," presented at the CLEO:2011 - Laser Applications to Photonic Applications (CLEO: S and I) Conference, Baltimore, MD, May 1-6, 2011, Paper CThBB2.

[2] Aijun Jin; Zefeng Wang; Jing Hou; Bin Zhang; Zongfu Jiang; , "Experimental measurement and numerical calculation of dispersion of ZBLAN fiber," Electronics and Optoelectronics (ICEOE), 2011 International Conference on, vol.3, pp.V3-181-V3-184, 29-31 July 2011

[3] L. G. Cohen "Comparison of Single-Mode Fiber Dispersion Measurement Techniques," Journal of lightwave technology, vol. 3, pp. 958 - 966, Oct. 1985

[4] S. Diddams, and J. C. Diels, "Dispersion measurements with white-light interferometry," Journal of Optical Society of America B, vol. 13, pp. 1120-1129, Jun. 1996.

[5] M. Tateda, N. Shibata, and S. Seikai, "Interferometric method for chromatic dispersion measurement in a singlemode optical fiber," IEEE Journal of Quantum Electronics, vol. 17, pp. 404-407, Mar. 1981.

[6] C. Lin, A. R. Tynes, A. Tomita, P. L. Liu, and D. L. Philen, "Chromatic dispersion measurements in single-mode fibers using picosecond InGaAsP injection lasers in the 1.2- to $1.5-\mu \mathrm{m}$ spectral region," Bell System Technical Journal, vol. 62, pp. 457-462, Feb. 1983.

[7] L. G. Cohen and C. Lin, "A universal fiber-optic (UFO) measurement system based on a near-IR fiber Raman laser," IEEE Journal of Quantum Electronics, vol. QE-14, pp. 855-859, Nov. 1978.

[8] J. Chang, H. Takahashi, I. Sugimoto, A. Oyobe, Mikio Ogai, "Measurement of chromatic dispersion with a GeO2 fiber Raman laser", presented at the Optical Fiber Communication Conference (OFC), San Diego, CA, Feb. 13-14, 1985, paper TUD3.

[9] Johan Hult, Rosalynne S. Watt, and Clemens F. Kaminski "Dispersion Measurement in Optical Fibers Using Supercontinuum Pulses," Journal of lightwave technology, Vol. 25, pp. 820-824, Mar. 2007.

[10] K. Mori, T. Morioka, and M. Saruwatari, "Group velocity dispersion measurement using supercontinuum picosecond pulses generated in an optical fibre," Electronics Letters, vol. 29, pp. 987-989, Nov. 1993.

[11] F. Koch, S. V. Chernikov, and J. R. Taylor, "Dispersion measurement in optical fibres over the entire spectral range from $1.1 \mu \mathrm{m}$ to $1.7 \mu \mathrm{m}$," Optics Communications, vol. 175, pp. 209-213, Feb. 2000.

[12] N. Nishizawa, A. Muto, and T. Goto, "Measurement of chromatic dispersion of optical fibers using wavelength-tunable soliton pulses," Japanese Journal of Applied Physics, vol. 39, pp.4990-4992, Aug. 2000. 
[13] Hen-Tai Shang, "Chromatic dispersion measurement by white-light interferometry on meter-length single-mode optical fibers," Electronics letters, vol. 17, pp. 603-605, Aug. 1981.

[14] B. Costa, D. Mazzoni, M. Puelo, and E. Vezzoni, "Phase-shift technique for the measurement of chromatic dispersion in optical fibers using LED's," IEEE Journal of Quantum Electronics, vol. 18, pp. 1509-1515 (1982).

[15] J.Y. Lee, and D.Y. Kim, "Versatile chromatic dispersion measurement of a single mode fiber using spectral white light interferometry," Optics Express, vol. 14, pp. 11608 - 11615, Nov. 2006.

[16] T. Zhang, Z. Yang, W. Zhao, Y. Wang, P. Fang, C. Li, "Dispersion measurement of Yb-doped fiber by a spectral interferometric technique," Chinese Optics Letters, 8, pp. 262-265, Mar. 2010.

[17] Q. Ye, C. Xu, X. Liu, W.H. Knox, M.F. Yan, R.S. Windeler, B. Eggleton, "Dispersion Measurement of Tapered Air-Silica Microstructure Fiber by White-Light Interferometry," Applied Optics, v. 41, pp. 4467-4470, Aug. 2002.

[18] P. Merritt, R. P. Tatam, and D. A. Jackson, "Interferometric Chromatic Dispersion Measurements on Short Lengths of Monomode Optical Fiber," Journal of lightwave technology, vol.7, pp. 703 - 716, Apr. 1989.

[19] E. Sorokin, N. Tolstik, and I. T. Sorokina, "Femtosecond operation and self-doubling of $\mathrm{Cr}: \mathrm{ZnS}$ laser," presented at the Nonlinear Optics: Materials, Fundamentals and Applications conference, Kauai, HI, June 17-22, 2011, paper NThC1.

[20] E. Sorokin, N. Tolstik, and I. T. Sorokina, "Kerr-Lens Mode-locked Cr:ZnS Laser", presented at Advanced Solid-State Photonics Conference (ASSP), San Diego, CA, Jan. 29 - Feb. 1, 2012, paper AW5A.5v

[21] V. Dvoyrin, I. Sorokina, V. Kalashnikov, V. Mashinsky, L. Ischakova, E. Dianov, V. F. Khopin, and A. N. Guryanov, "Tm3+-doped CW fiber laser based on a highly GeO2-doped dispersion shifted fiber," presented at the in Advanced Solid-State Photonics Conference (ASSP), Istanbul, Turkey, February 13-16, 2011, paper ATuB17.

[22] S. H. Wemple, "Material Dispersion in Optical Fibers," Applied Optics, vol. 18, pp. 31-35, Jan. 1979.

[23] V. L. Kalashnikov, "Effective refractive indexes and dispersion characteristics of the tapered fibers," Available: http://info.tuwien.ac.at/kalashnikov/TFmodes.html. 